\title{
¿Quién cura y cómo cura? Tratamiento de la enfermedad en una comunidad rural de Ecuador
}

\author{
Who heals and how does it cure? Treatment of the \\ disease in a rural community from Ecuador \\ Quem cura e como se cura? Tratamento da doença em \\ uma comunidade rural do Equador
}

\begin{abstract}
Jessica Valeria Pasto-Capuz ${ }^{1}$, Judith Inmaculada Francisco Pérez², Manuel Ramón Blanco ${ }^{3}$

${ }^{1}$ Estudiante de la Carrera de Enfemería de la Universidad Técnica de Ambato tFalleció inesperadamente en febrero de 2018. Para esa fecha, ya había recopilado todas las entrevistas y había transcrito las mismas. Colaboró igualmente en la búsqueda de bibliografía. Era nuestra alumna de titulación. En reconocimiento a su compromiso con el trabajo, es la primera autora. Dedicación a su memoria ${ }^{2}$ PhD. Educación a Distancia. Pontificia Universidad Católica del Ecuador ${ }^{3}$ Médico, Magíster en Salud Pública. Pontificia Universidad Católica del
\end{abstract} Ecuador

\begin{abstract}
Cómo citar este artículo en edición digital: Pasto-Capuz, V., Francisco-Pérez, J. \& Blanco, MR. (2019). ¿Quién cura y cómo cura? Tratamiento de la enfermedad en una comunidad rural de Ecuador. Cultura de los Cuidados (Edición digital), 23 (54). Recuperado de http://dx.doi.org/10.14198/cuid.2019.54.21

Correspondencia: Mariscal Foch y José Tamayo. N. 10-37. Parroquia Mariscal Sucre. Quito. CP. 170123

Correo electrónico de contacto: judithfrancisco@gmail.com

Recibido: 29/01/2019; Aceptado: 12/05/2019
\end{abstract}

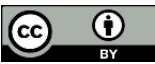

\section{ABSTRACT}

Introduction: Traditional medicine is a therapeutic alternative that respects ancestral knowledge and multiculturalism.

Objective: To explore the practices used for the cure of diseases in the rural community of San Miguel de Chalata, Ecuador.

Methods: A qualitative, hermeneutical research was carried out. A semi-structured interview was answered by 22 older adults living in the community. The discourses were recorded, transcribed and analyzed.

Results: Traditional medicine is valued to cure somatic diseases due to its accessibility, low cost and effectiveness. They recognize conventional medicine in the cure of chronic diseases, however, they associate it with prolonged waiting times and discriminatory treatment with the elderly. They use rituals in supernatural diseases, mixing faith, religiosity and assistance of the universal divine power.

Conclusions: Traditional and conventional medicine have defined attributions in the collective thought of this community. They approve the power of the healer and respect the scientific knowledge of professional 
doctors. The barriers of the conventional system should be studied because of their importance in the use of health services and equality in health.

Keywords: Traditional medicine, cultural diversity, alternative medicine.

\section{RESUMO}

Introdução: A medicina tradicional é uma alternativa terapêutica que respeita o conhecimento ancestral e o multiculturalismo.

Objetivo: Explorar as práticas utilizadas para a cura de doenças na comunidade rural de San Miguel de Chalata, Equador.

Métodos: Uma pesquisa qualitativa e hermenêutica foi realizada. Responderam a uma entrevista semiestruturada 22 idosos vivendo na comunidade. Os discursos foram gravados, transcritos e analisados.

Resultados: A medicina tradicional é valorizada para curar doenças somáticas, devido à sua acessibilidade, baixo custo e eficácia. Eles reconhecem a medicina convencional na cura de doenças crônicas. No entanto, associam-na a prolongados tempos de espera e tratamento discriminatório com os idosos. Eles usam rituais em doenças sobrenaturais, misturando fé, religiosidade e assistência do poder divino universal.

Conclusões: A medicina tradicional e convencional têm atribuições definidas no pensamento coletivo dessa comunidade. Eles aprovam o poder do curador e respeitam o conhecimento científico dos médicos profissionais. As barreiras do sistema convencional devem ser estudadas devido à sua importância no uso de serviços de saúde e igualdade na saúde.

Palavras chave: Medicina tradicional, diversidade cultural, medicina alternativa.

\section{RESUMEN}

Introducción: La medicina tradicional es una alternativa terapéutica que respeta los saberes ancestrales y la multiculturalidad.

Objetivo: Explorar las prácticas utilizadas para la curación de enfermedades en la comunidad rural San Miguel de Chalata, Ecuador.

Métodos: Se realizó una investigación cualitativa, hermenéutica. Respondieron una entrevista semiestructurada, 22 adultos mayores residentes en la comunidad. Los discursos fueron grabados, transcritos $y$ analizados.

Resultados: Se valora a la medicina tradicional para curar enfermedades somáticas por su accesibilidad, bajo costo $\mathrm{y}$ efectividad. Reconocen a la medicina convencional en la curación de enfermedades crónicas. Sin embargo, la asocian con prolongados tiempos de espera y trato discriminatorio con las personas de mayor edad. Usan rituales en enfermedades sobrenaturales, mezclando fe, religiosidad y asistencia del poder divino universal.

Conclusiones: La medicina tradicional y la convencional tienen atribuciones definidas en el pensamiento colectivo de esta comunidad. Aprueban el poder del sanador y respetan el conocimiento científico de los médicos profesionales. Las barreras del sistema convencional deberían estudiarse por su importancia en la utilización de los servicios sanitarios y la igualdad en salud.

Palabras clave: Medicina tradicional, diversidad cultural, medicina alternativa.

\section{INTRODUCCIÓN}

La medicina tradicional es valorada mundialmente como tratamiento de fácil acceso y con menos efectos adversos que la medicina convencional (OMS, 2013; OMS, 
2003). En países de Asia y Europa se reconoce como una alternativa que respeta la multiculturalidad (TCM Clinic, 2003). En Estados Unidos existe una alta demanda de plantas para el manejo terapéutico de diversas sintomatologías (Morón, 2012).

En Latinoamérica existe interés científico en esta medicina por su penetración cultural y carácter ancestral, basada en el poder de la naturaleza y del curandero, quien invoca a las energías supremas mediante rituales de sanación (OPS/OMS, 2002). Por ello, recomiendan introducir su estudio en la formación de profesionales de la salud (Cruz, Tase, Martínez y Moneja, 2015; Guillaume, Quintero, Estrella, Morales y Matos, 2012).

En México, parte de la población resuelve sus enfermedades agudas mediante plantas, requiriendo asistencia médica convencional en casos de gravedad (Gómez, 2012). Generalmente son los adultos mayores quienes curan afecciones causadas por energías provenientes de otras personas (White-Olascoaga, Juan-Pérez, ChávezMejía y Gutiérrez-Cedillo, 2013). Al igual que en Colombia, en otros lugares los curanderos estabilizan espiritualmente al enfermo, siendo sus intermediarios con la naturaleza, donde reside el poder de sanación (Cardona y Rivera, 2012; López, Castaño, López y Velásquez, 2011). Es importante la fe del paciente, especialmente en enfermedades avanzadas como el cáncer (Venegas et al., 2008).

Ecuador preserva diversas prácticas ancestrales en la curación de la enfermedad (White-Olascoaga et al., 2013). En algunas poblaciones existen chamanes, quienes diagnostican y curan utilizando palabras, movimientos, animales, plantas y objetos representativos del mundo espiritual. Por ello, la Constitución Nacional del país establece el respeto a la medicina tradicional y los saberes ancestrales de sus comunidades indígenas (Cruz et al., 2015).

Bolívar es una provincia de la sierra ecuatoriana habitada por indígenas $\mathrm{y}$ mestizos, con una elevada riqueza cultural. Allí se encuentra San Miguel de Chalata, una comunidad rural de 600 habitantes, quienes utilizan la medicina ancestral para curar sus problemas de salud (Cruz et al., 2015).

La investigación tuvo el propósito de explorar las prácticas utilizadas para la curación de enfermedades en esta comunidad rural. Una forma de reconocer su existencia y generar conocimientos para promover relaciones más justas, éticas $y$ humanas entre personas de diferentes grupos culturales (Falkenberg, Shimizu \& Bermudez (2017).

\section{MÉTODOS}

Se realizó una investigación cualitativa, hermenéutica, insertada dentro del paradigma interpretativo. Este estudio no generaliza resultados y está orientado al entendimiento de las experiencias de las personas con relación al fenómeno en estudio, basado en la interpretación de sus los argumentos (Hernández, Fernández y Baptista, 2010).

Participaron 22 adultos mayores, seleccionados intencionalmente por practicar la medicina tradicional. Se utilizó la entrevista semiestructurada, conformada por cinco preguntas abiertas. Las entrevistas fueron grabadas y literalmente transcritas. Posteriormente, se construyó una tabla con el contenido del discurso y se analizó, identificando en cada línea, conceptos o ideas que funcionaron como códigos. Estos se agruparon diferenciando la coincidencia de los discursos, dando origen a las categorías emergentes de análisis. Las categorías se analizaron en un proceso de 
triangulación, sustentado en la bibliografía relacionada con el tema y los antecedentes de otras investigaciones similares. De la triangulación surgieron las conclusiones del estudio.

\section{Aspectos éticos}

Se consideraron las recomendaciones de Gordillo (2012) en cuanto a los principios para el manejo de información. Se presentó el estudio al presidente de la comunidad. Se entregó a informantes un documento con las características del estudio, implicaciones, beneficios, riesgos, confidencialidad $y$ compromisos de las investigadoras por culminar el estudio y publicar los resultados. Se adjuntó el consentimiento informado, dando dos días a las personas para su consideración y aceptación.

\section{RESULTADOS}

El análisis de los discursos permitió identificar 32 códigos asociados con problemas de salud tratados en la comunidad. Este análisis y la similitud entre los códigos identificados, permitió organizar conceptos más amplios, conformando las categorías emergentes que se muestran en la Figura 1.

FIGURA 1: Categorías emergentes identificadas en los discursos

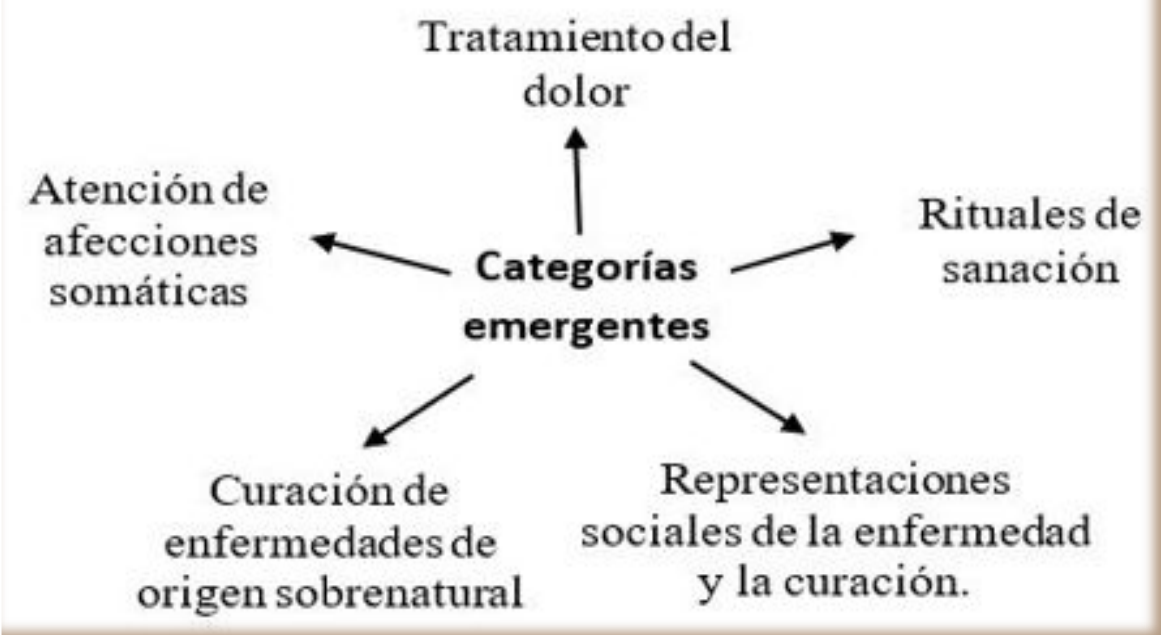

Fuente: Análisis de los discursos. Pasto y Francisco (2017)

Se identificaron cinco categorías emergentes, tituladas: tratamiento del dolor, atención de afecciones somáticas, curación de enfermedades de origen sobrenatural, rituales de sanación y representaciones sociales de la enfermedad y la curación. Los códigos quedaron distribuidos por categorías de la siguiente manera: cuatro en la primera, 10 en la segunda, ocho en la tercera, dos en la cuarta y cinco en la quinta. A partir de este momento del estudio, se analizó separadamente cada categoría, las cuales se describen en las figuras 2 a la 6. 


\section{Cultura de los Cuidados}

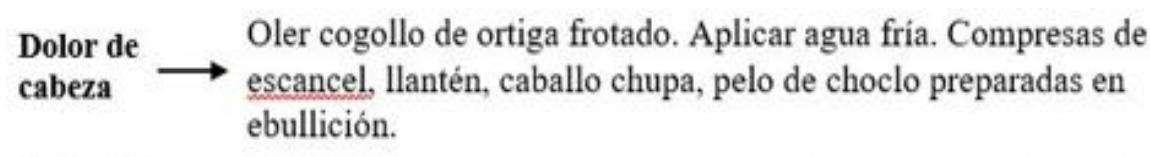

Dolor del
corazón $\longrightarrow \begin{aligned} & \text { Ingerir varias tomas de agua fria preparada con dos cogollos de } \\ & \text { toronjil machucado con las manos, previamente lavados }\end{aligned}$

Dolor en $\longrightarrow \begin{aligned} & \text { Pasar ortiga por las piernas. Bañarse o lavarse las piernas con } \\ & \text { piernas }\end{aligned}$
agua caliente de ortiga hervida, manzanilla o matico.

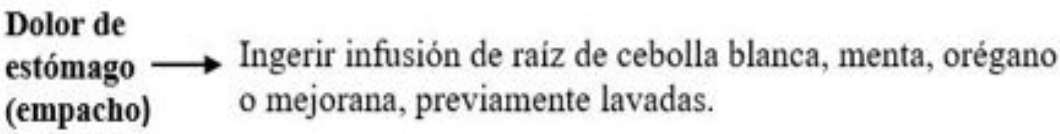

FuENTE: Análisis de los discursos. Pasto y Francisco (2017)

Tal como se observa en la Fig. 2, los informantes tratan el dolor utilizando infusiones de plantas por vía oral. Igualmente, usan la vía inhalatoria de algunas plantas frotadas, aplican compresas directamente sobre la zona adolorida y emplean agua de hierbas cocidas como tópico o baño corporal.

\section{FIGURA 3: Curación de afecciones somáticas}

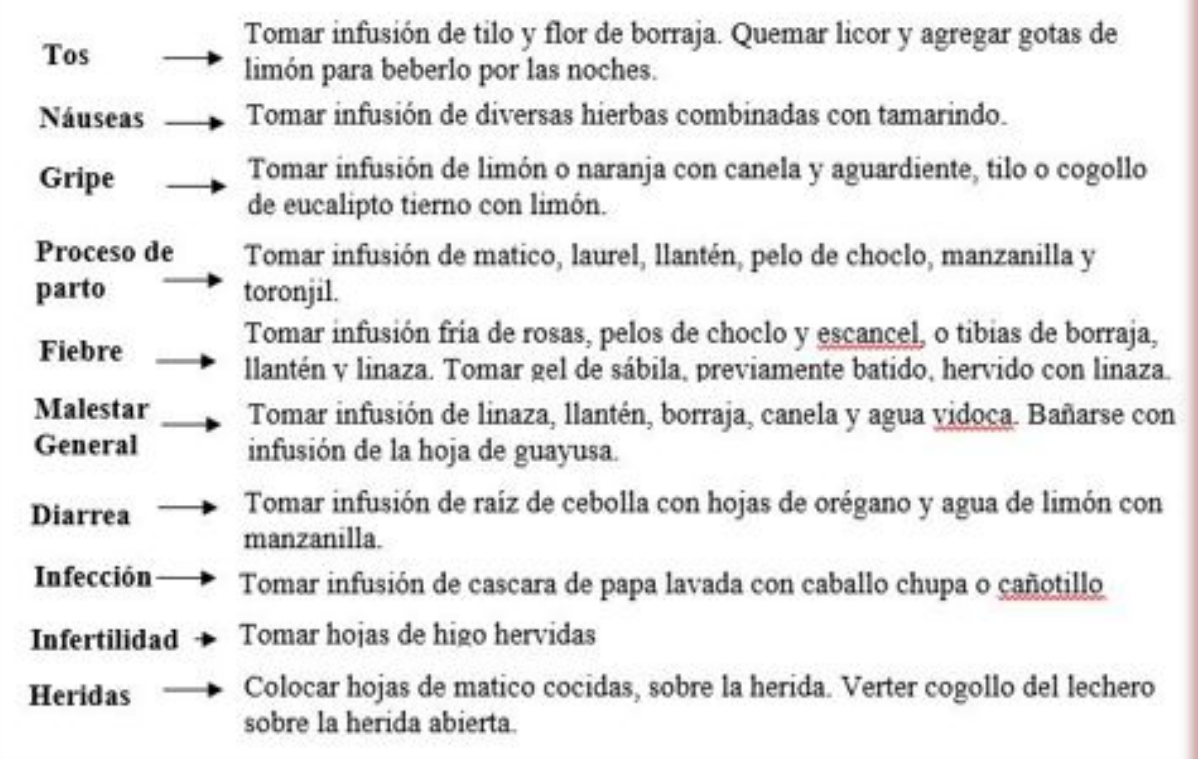

FuENTE: Análisis de los discursos. Pasto y Francisco (2017)

En la Fig. 3 se muestran las distintas terapias empleadas en la curación de enfermedades somáticas. En casi todas usan infusiones $y$ en menos casos, plantas 
hervidas y en zumo. También colocan heridas. hierbas directamente en la piel para curar las

\section{FIGURA 4: Medidas no farmacológicas utilizadas en la curación de enfermedades de origen sobrenatural}

\footnotetext{
Espanto $\longrightarrow$ Sacudir sobre el cuerpo con manojo de hierbas, previamente calentadas al fuego humedecidas con perfume v humear el ambiente con las hoias quemadas.

Mal de oio $\rightarrow$ Limpiar el cuerpo con montes. Ingerir orina mezclada con hoias de freiol verde.

Pachanchi $\rightarrow$ Ingerir orina mezclada con manteca de cacao. Hervir un pedazo de fierro, usar

Maligno $\longrightarrow$ Limpiar el cuerpo con montes calentados en el fuego y humear con cigarrillo. Beber infusión de raíz de cebolla blanca, congona y ruda.

Mal bajo $\rightarrow$ Frotar dos huevos de gallina por el cuerpo, junto con montes de viento (santa maría, mashuwa negra y blanca, pumin, quila, entre otros), soplar con licor y perfume. Frotar una gallina culeca por el cuerpo.

Colerín $\rightarrow$ Beber infusión de cogollo de toroniil cernido con limón.

Bruiería $\rightarrow$ Encargado a los chamanes que poseen objetos sagrados y especies personales como preparados, piedras y hierbas especiales.

Mal aire $\rightarrow$ Usar un manojo de plantas especificas para rociar con perfume. A la par, humar con cigarrillo. Pasar dos huevos por el cuerpo y luego descartar.
}

FuENTE: Análisis de los discursos. Pasto y Francisco (2017)

Tal como se describe en la Fig. 4, se identificaron ocho enfermedades de origen sobrenatural. Para su curación utilizan el humo de plantas quemadas, orina humana, humo de cigarrillo e infusiones. En casos particulares como en el mal bajo, frotan huevos de gallina por el cuerpo, soplan con licor y agregan perfume. La brujería, es atendida por el chamán mediante objetos sagrados y especies personales.

FIGURA 5: Rituales empleados durante y después del acto de sanación

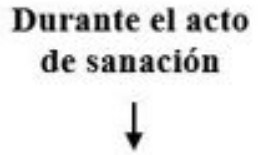

Para el diagnóstico se recorre el cuerpo con el cuy y abrir el animal para visualizar internamente. Sus órganos reflejarán las enfermedades del individuo. Encender una vela y pedir al paciente que infunda su esencia soplándola. El estado de la llama expresa su estado de salud.

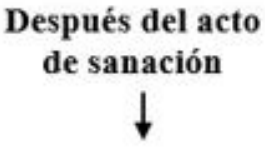

Restricción de comidas irritantes pues anulan la limpieza realizada. Al cuarto día bañarse con agua de rosas y claveles blancos y rojos, combinado con limón, naranja, piña y ruda. Volver al octavo dia, para repetir parte del ritual y verificar la evolución del individuo.

FueNTE: Análisis de los discursos. Pasto y Francisco (2017)

Tal como se describe en la Fig. 5, los informantes realizan rituales conducidos 


\title{
Cultura de los Cuidados
}

por personas conocedoras de la medicina andina, llamados Yachak o sabios ancestrales. En el acto de sanación utilizan el cuy, un roedor que permite curar y diagnosticar la enfermedad absorbiendo en sus propios órganos todos los males de la persona enferma. Con posterioridad a la sanación, se incluyen restricciones alimentarias y baños preparados de hierbas.

\section{FIGURA 6: Representaciones sociales de la enfermedad y el proceso de sanación}

\begin{abstract}
Atribuciones de
la medicina

convencional

"....si el médico del campo dice...., tengo que ir al médico del centro de salud" (II)) "me hago tratar con el doctor porque no mejoraba" (121) "tengo problemas en los huesos, para eso no puedo tomar remedios naturales" (I14)

Barreas de la medicina convencional

" upara qué ir donde el doctor?, ya por la edad no $\longrightarrow$ quieren ni verlo a uno" (I2) "...el trato no es el mismo con nosotros los viejos" (14) "no somos preparados como ellos, a veces ellos no entienden" (I5) "... la medicina que mandan es buena, pero es cara y también pasan muchos meses para poderse ver" (I6)

Atribuciones a la "ya no se gasta en medicamentos de las boticas" (I8). "La Medicina medicina tradicional es buena porque es natural y Tradicional $\longrightarrow$ curativa" (I6) "...la medicina natural es más sana" (I20) "Hay que saber recoger para qué es, asi se puede curar" (13) "Todo lo que tenemos en el campo es remedio (118).

Curación

"Todo depende de la fe" (II) "...hay que tener fe y se por fe siente mejoria" (I3) "...pedir a Dios con fe que salga todo lo malo" (14) "Si se compone uno es por la fe, por voluntad" de Dios" (110) "...creer ...si no tiene es a fe tampoco se cura uno" (114) "He creido en esos chamanes cuando estaba enfermo y me curi"ind (122)

Origen del muestros ancianos conocian la preparación de estos
conocimiento $\longrightarrow \begin{gathered}\text { remedios" (I7) "wlas personas mayores han sabido curar y } \\ \text { sanador }\end{gathered}$
aprendimos de ellos" (19) "andando con la gente mayor, que
sabe cosas, se aprende mejor" (110) "Nuestros padres y
abuelos, asi sabian curar, nosotros aprendimos con ellos" (I8)
\end{abstract}

FuENTE: Análisis de los discursos. Pasto y Francisco (2017)

Tal como se muestra en la Fig. 6, se agruparon cuatro códigos referidos a las atribuciones de la medicina convencional, atribuciones de la medicina tradicional, la curación por fe y el origen del poder sanador. La medicina tradicional es la primera forma de tratamiento para la curación de enfermedades somáticas y de tipo sobrenatural. Se reconoce su accesibilidad, su efecto inmediato y bajo costo. El poder del sanador proviene del conocimiento ancestral, transmitido de forma directa por los curanderos de la comunidad. La fe en el ser supremo, el curandero y el tratamiento a utilizar, tiene un papel determinante en la curación.

Se valora a la medicina convencional en la atención de enfermedades crónicas. El 
sistema sanitario tiene barreras percibidas como trato desigual, retardo en el acceso a la atención y de comunicación.

\section{DISCUSIÓN DE RESULTADOS}

Las afecciones de origen somático se tratan con plantas medicinales como manzanilla, menta, toronjil, taraxaco, sábila, entre otras, las que son de fácil acceso y producción espontánea en los hogares. La mayoría de las personas las utiliza para aliviar manifestaciones físicas como el dolor, la fiebre y el malestar (Cruz et al., 2015; Gómez, 2012). Incluso en cáncer, donde algunos pacientes usan hierbas, oraciones y sustancias especiales que ayudaron a ralentizar el proceso de metástasis (Venegas et al., 2008; Valdez-Cobo, 2013; Czubaj, 2005).

Se reconoce a la medicina convencional en la curación de afecciones somáticas cuando son crónicas $\mathrm{o}$ han ocasionado complicaciones, similar a otros casos en donde las personas no mejoraron usando medicina natural (Gómez, 2012). Sin embargo, la asocian con comunicación impersonal $y$ trato discriminatorio, especialmente en personas mayores. Esto pudiera afectar la búsqueda de atención médica y la continuidad del cuidado de estos grupos minoritarios (Crawley, Ahn \& Winkleby, 2008; Regts, 2013; Ortíz, Baeza, Salinas, Flynn \& Betancourt, 2016).

El modo de preparación más sobresaliente es la infusión, aunque también usan zumos, jugos, aguas frescas y aguas reposadas, a veces combinados con licor caliente administrados por vía oral. De manera similar otras investigaciones aquí también se considera que las propiedades curativas de las hojas se manifiestan con la cocción (Angulo, Rosero y González, 2012; Bernal, López, Murilla \& Méndez, 2015; Barrragán-Solis, 2006).

Algunas afecciones manifestadas en la piel se tratan con ungüentos, compresas y aplicación de hojas. De ello se puede interpretar que reconocen a la piel como una vía de administración donde se absorben los principios activos, necesarios para la curación.

Los informantes han construido saberes para identificar, diagnosticar y tratar algunas enfermedades de origen sobrenatural, las que tienen en común el ser causadas por otras personas, directa o indirectamente. Son de aparición súbita y frecuente en la comunidad, causan debilidad progresiva y pueden comprometer la vida del afectado según la magnitud de las energías negativas que estén involucradas.

La brujería es la única enfermedad encargada exclusivamente a los chamanes. Ellos conocen el tratamiento con preparados especiales, objetos sagrados, representaciones divinas, piedras y una variedad de hierbas disponibles en la comunidad. Además, conocen los días donde abunda la energía positiva requerida para que ocurra la sanación. En ese sentido, hay coincidencia con otras investigaciones, en donde se considera al chamán el único encargado de los ritos, rezos, plegarias, prácticas mágicas e invocaciones necesarias para devolver la salud, porque tiene el poder para actuar en armonía con la energía divina para alejar la maldad que causa la enfermedad (Barragán-Solís, 2006; Vicente, Rojas, Vivanco y Vallejo, 2011; Gubler, 1996; Pereyra y Guzmán, 2010).

En los pueblos donde se practica la medicina tradicional se reconoce socialmente a los curanderos, cuya sabiduría proviene de Dios (Magaña, 2010; Henao, 2006) aceptando su capacidad para formar a otras personas en un proceso informal e integral (López et al., 2011). Los rituales siguen protocolos específicos de sanación, 
tal como otros estudios donde han fijado plantas y días concretos donde abunda la energía que la persona requiere para recuperar la integridad del alma y eliminar la enfermedad (Gubler, 1996).

Un ritual muy utilizado es la limpia mediante el cuy, un roedor que, al ser frotado por el cuerpo del enfermo, absorbe la enfermedad, permitiendo la curación. Un estudio previo describe el poder mágico de este ritual, que incluye el sacrificio posterior del animal para ver en sus órganos las afectaciones que le fueron retiradas a la persona tratada (Bianchetti, 2014).

La fe es un elemento representativo en el tratamiento tradicional de esta comunidad. La persona debe creer en el curandero y en la divinidad que le otorga el poder de curar, pues influye en la salud y la enfermedad (Duque y Barco, 2015; Charcot, 2001).

\section{CONCLUSIONES}

Los informantes reconocen enfermedades somáticas coincidentes con patologías de la nosografía convencional e identifican enfermedades de origen sobrenatural en las cuales la medicina convencional no puede actuar. Tienen como primera elección el uso de medidas no farmacológicas para la atención de sus problemas somáticos de manifestación aguda.

La medicina tradicional y la convencional, tienen atribuciones claramente definidas en el pensamiento colectivo de esta comunidad. Aprueban el poder sanador de los médicos tradicionales y respetan el conocimiento científico de los médicos profesionales, cada uno en particulares alteraciones de la salud. Usan la medicina casera en el tratamiento de la mayoría de sus afecciones, pero reconocen la propiedad de la medicina convencional para la atención de enfermedades crónicas. En los adultos mayores reside la transferencia de saberes ancestrales para atender padecimientos somáticos mediante el uso de plantas medicinales. Actúan de manera similar a un sistema de atención primaria dentro de su propia comunidad. Reciben al enfermo, diagnostican el problema y ofrecen tratamiento administrado principalmente por vía oral y tópica. La medicina natural no tiene efectos adversos, es más económica y más accesible. Se reconoce al tratamiento tradicional en la curación de enfermedades de origen sobrenatural, por sus beneficios corporales y espirituales. El médico tradicional trata desde afuera, para curar desde adentro mediante rituales de sanación. Es intermediario entre la persona enferma y el poder divino sanador, usando fricción con plantas, humo de cigarrillo, sahumerio, licor, flores y preparados con esencias.

Los habitantes de esta comunidad poseen saberes socialmente construidos que determinan sus prácticas de salud. Su interacción con los profesionales del sistema convencional debería ser estudiada por ser una condición para mejorar la utilización de los servicios sanitarios, el control de padecimientos crónicos y reducir las desigualdades en salud. Así, ambos sistemas podrían complementarse en concordancia con las políticas de la nación, en beneficio de la población.

\section{Responsabilidades éticas}

La autora declara que para esta investigación no se realizaron experimentos con seres humanos y animales. No se indican datos de los informantes, pues se respetaron todos los principios relacionados con la privacidad de la información y el consentimiento informado de los participantes y no se recibieron fondos institucionales ni de otra ninguna organización para realizar el estudio.

\section{BIBLIOGRAFÍA}

- Barragán-Solis, A. (2006). La práctica de la 
autoatención por fitoterapia en un grupo de familias mexicanas. Arch Med Fam, 8(155-162). Consultado en http://www.medigraphic.com/pdfs/medfam/amf2006/amf063c.pdf.

- Bernal, J. M, López, A. F., Murilla, E. \& Méndez, J. J. (2015). Flora silvestre medicinal utilizada por los Kofan colombianos en el tratamiento de la leishmaniasis cutánea. Revista Cubana de Plantas Medicinales, 19(4). Consultado en http://www.revplantasmedicinales.sld.cu/index.php Lpla/article/view/142/87.

- Bianchetti, M. C. (2014). Curanderos, especializaciones y afecciones que rigen aún hoy en el área andina centro oeste suramericano. Scripta Ethnologica, XXXVI, 129-164. Consultado en http://www.redalyc.org/articulo.oa?id=14832692005.

- Cardona, J. A \& Rivera Palomino, Y. (2012). Representaciones sociales sobre medicina tradicional y enfermedades foráneas en indígenas Embera Chamí de Colombia. Revista Cubana de Salud Pública, 38(3), 471-483. Consultado en https://bit.ly/2FOCZOW.

- Crawley, L. M, Ahn, D. K, Winkleby, M. A. (2008). Perceived medical discrimination and cancer screening behaviors of racial and ethnic minority adults. Cancer Epidemiol Biomarkers Prev, 17(8): 193744.

- Czubaj F. (2005). Eligen terapias alternativas para atacar el cáncer. La Nación; Buenos Aires [Internet]. Consultado en https://bit.ly/2R7BgFr.

- Charcot, J. M. (2001). La fe que cura. Revista de la Asociación Española de Neuropsiquiatría, (77), 99-111. Consultado en https://bit.ly/2S5P9Uw.

- Duque, J. E, Barco, J. (2015). La ciencia médica versus creencia en dios: la conservación del mito. Arch Med Col, 15(1). Consultado en https://bit.ly/2zosThI.

- Escalona, L. J., Tase, A., Estrada, A., Almaguer, M. L. (2015). Uso tradicional de plantas medicinales por el adulto mayor en la comunidad serrana de Corralillo Arriba. Guisa, Granma. Revista Cubana de Plantas Medicinales, 20(4) Consultado en https://bit.ly/2PQh0eR.

- Falkenberg MB, Shimizu HE, Bermudez XPD. (2017). Representaciones sociales de la atención sanitaria de la población indígena Mbyá-Guaraní por parte de trabajadores de salud. Rev. Latino-Am. Enfermagem, 25:e2846. Consultado en http://www.scielo.br/pdf/rlae/v25/es_0104-1169-rlae25-02846.pdf.

- Felipe Angulo, A., Rosero, R. \& González Insuasti, M. S. (2012). Estudio etnobotánico de las plantas medicinales utilizadas por los habitantes del corregimiento de Genoy, Municipio de Pasto, Colombia Ethnobotanical study of medicinal plants used by the inhabitants of the village of Genoy,
Municipality of Pasto, Colombi. Universidad y Salud, 14(2). Consultado en http://revistas.udenar.edu.co/index.php/usalud/artic le/view/1277.

- Gómez Álvarez, R. (2012). Plantas medicinales en una aldea del estado de Tabasco, México. Revista fitotecnia mexicana, 35(1), 43-49. Consultado en https://bit.ly/2Rds02r.

- Gordillo, A., Medina, U., Pierdant, M. (2012). Manual de investigación clínica. Mexico: Manual Moderno.

- Gubler, R. (1996) El papel del curandero y la medicina tradicional en Yucatán. Alteridades, 6(12): 11-8. Consultado en http://www.redalyc.org/articulo.oa?id=74711374003.

- Guillaume, V., Marín, M. E., Morales, E., Matos, N. (2012). Conocimiento y aplicación de la medicina natural y tradicional por profesionales y técnicos de la salud. Revista Cubana de Estomatología, 49(2), 89-98. Consultado en https://bit.ly/2AisLA9.

- Henao, J. (2006). La Fe Mueve Montañas. The Santa Fe New Mexican; Santa Fe, NM [Internet]. Consultado en https://bit.ly/2KxyVks.

- Hernández, R., Fernández, P., y Baptista, L. (2010). Metodología de la Investigación. Quinta Edición. Mexico: Mc Graw Hill.

- López, L., Cataño, N., López, H., Velásquez, V. (2011). Diversidad cultural de sanadores tradicionales afrocolombianos: preservación y conciliación de saberes. Aquichan Chia, 11(3):287-304. Consultado en https://bit.ly/2r5ytBj

- Magaña, M. A., Gama, L. M., Mariaca, R. (2010). El uso de las plantas medicinales en las comunidades Maya-Chontales de Nacajuca, Tabasco, México. Polibotánica, (29), 213-262. Consultado en https://bit.ly/2KuTYnS.

- Morón, F. (2012). Las plantas medicinales como recurso de salud de bajo costo para el adulto mayor. Revista Cubana de Plantas Medicinales, 17(4), 297-299. Consultado en https://bit.ly/2S9mT3t.

- Organización Mundial de la Salud. (2003). Medicina Tradicional [Internet]. Consultad en https://bit.ly/2Bumh2S.

- Organización Mundial de la Salud. (2013). Estrategia de la OMS sobre medicina tradicional 2014 - 2023 [Internet]. OMS; Consultado en https://bit.ly/2THFCVm.

- Organización Panamericana de la Salud/Organización Mundial de la Salud. (2002). Estrategia de la OMS sobre Medicina Tradicional 2002-2005.

Consultado en http://www.ops.org.bo/textocompleto/pi31676.pdf.

- Ortiz, M. S., Baeza-Rivera, M. J., Salinas-Oñate, N., Flynn, P., Betancourt, H. (2016). Atribución de malos tratos en servicios de salud a discriminación y sus consecuencias en pacientes diabéticos mapuche. Rev 
Med Chile, 144(10): 1270-6. Consultado en: https://bit.ly/2DZdwAq.

- Pereyra, R.G., Guzmán, E.R. (2010). Curanderismo y magia: Un análisis semiótico del proceso de sanación. CULCyT Cult Científica Tecnológica, (38): 5-15. Consultado en https://dialnet.unirioja.es/servlet/articulo?codigo $=37$ 39085.

- Regts, G.J. (2013). "Discrimination-Based Attributions for Mistreatment Affect Continuity of Care". Loma Linda University Electronic Theses, Dissertations \& Projects. $\quad 147 . \quad$ Consultado en http://scholarsrepository.llu.edu/etd/147.

- TCM Clinic. (2003). Medicina china: Regulación en Europa-[Internet]. Consultado en https://bit.ly/2THxdRI.

- Valdes-Cobos, A. (2013). Conservacion y uso de plantas medicinales: el caso de la región de la Mixteca Alta Oaxaquena, Mexico. Ambiente y Desarrollo, 17(33), 87-97.
- Venegas, V., Chaves, M., Beltran, G., Díaz, G., Estrada, M., Gonzalez, Y., Muñoz, A. (2008). Creencias populares acerca de la curación del cáncer de próstata y cérvico uterino y experiencias de aplicación en pacientes de una institución de salud, Bogotá. Revista Colombiana de Enfermería. Vol 3, Año 3, 69-76. Consultado en https://bit.ly/2NCLVIQ.

- Vicente, S., Rojas, R., Flores, Vivanco, K., Vallejo, M. (2011). Recuperación histórica del patrimonio cultural de salud en la región sur de Ecuador y norte del Perú Loja-Piura. Pimera Edición. Loja-Piura: Imprenta Santiago. 398 pp.

- White-Olascoaga, L., Juan-Pérez, J. I., Chávez-Mejía, C., Gutiérrez-Cedillo, J. G. (2013). Flora medicinal en San Nicolás, municipio de Malinalco, Estado de México. Polibotánica, (35), 173-206. Consultado en https://bit.ly/2Qdqi47. 